\title{
Research and Implementation of Plotting Based on MapX TrueType Font Symbol
}

\author{
Yongzhen Qin*, Quanxing Miao, Renyuan Hu and Hu Zhao \\ Engineering University of Armed Police Force, Xi'an, China \\ ${ }^{*}$ Corresponding author
}

\begin{abstract}
Plotting on the electronic map be of great importance and widely used. The method to plot regular unit symbol on map with MapX TrueType font symbol is researched, and the way is realized using MapX and Visual $\mathrm{C}++$. It is showed that the method is more effective and has good quality of reality and real-time and good practical effect. and also has definite value to make popular.
\end{abstract}

\section{Keywords-MapX; plotting; TrueType font symbol}

\section{INTRODUCTION}

Plotting which Puts unit Symbols on Electric Maps is basic function of many geographic information systems, so it is a key question of the computer aided plotting system and is researched for long time. Unit symbols may be regular symbols or irregular symbols. Regular symbols are important symbols in the computer aided plotting system, their dimensions such as length and width of the symbols always scale up or scale down as its size varies.

MapInfo MapX is a mapping control with powerful mapping capabilities, it may be added to a new application or the old one. MapX which is based on the same mapping technology used in other MapInfo products, such as MapInfo Professional, is powerful. With MapX, you can analyze and visualize your data, create or edit map features, and display the results geographically. Key features of MapX are Thematic mapping, Drill-down mapping, Data binding, Annotations, Layering, Raster Images, Automatic Labeling, Selections, Feature Factory, Tools, Map Editing, Projections and Coordinate Systems, Remote Spatial Server Connectivity[1]. MapX is effective and widely used to develop some geographic information systems and computer aided plotting systems[2-5].

The rest of paper is as follows: Section 2 describes the Method Plotting Based on MapX TrueType Font Symbol, and Section 3 describes the Implement with MapX and Visual C++, finally, Section 4 concludes the paper.

\section{TRUeTyPe FONT AND PlOtTING}

Maps in MapX are made up of layers of map objects. These map objects are accessed in MapX through the Feature object. There are four basic types of features such as region, point, line and text. Point objects represent single locations of data. For example, customer locations, restaurants, and parking meters. Point objects include bitmap symbols, TrueType font symbols and vector symbols. You can have each type of object to a separate layer (most common), or you can combine objects to the same layer. MapX lets user create, edit, customize, and display these objects to make maps that meet their needs.
MapX provides three ways for developer to directly create, edit, erase, customize, and display Point objects: bitmap symbols, TrueType font symbols and vector symbols. TrueType font symbols may be rotated, bitmap symbols and vector symbols are not be rotated. Bitmap symbols, TrueType font symbols and vector symbols are both not zoom in or zoom out automatically with the maps.

\section{A. TrueType Font Symbols of MapX}

MapX also uses some TrueType fonts. In MapX the TrueType fonts work As Feature object. With the TrueType font symbols, user may add many regular unit symbols on the maps, and edit, display them. As the layers objects, the properties such as background color, symbol color, halo, the number of degrees to rotate a TrueType symbol of the regular unit symbols may be controlled. The method is suitable to plot the regular unit symbols on maps.

\section{B. Plotting Based on TrueType Font}

The method to add the regular unit symbols on the maps with the TrueType font symbols should be work as following:

First, Before the regular unit symbols be added to the maps, they should be the characters of some font files. These font files must be copied to Window's Font Folder. Fonts must also be registered with Windows. If you are using a third-party software package to create your installer, that package might handle font registration for you. Otherwise, you can register fonts manually, by calling the Win32 Add Font Resource routine. Once a font is properly registered, the registry contains a corresponding key under:

- HKEY_LOCAL_MACHINE SSOFTWARE $\backslash$ Microsoft $\backslash$ Windows $\backslash$ CurrentVersion\Fonts

- HKEY_LOCAL_MACHINE\SOFTWARE\Microsoft $\backslash$ Windows NT\CurrentVersion\Fonts

Second, Style. Symbol Type property should be used to specified the Point Styles, i.e. set features to TrueType font symbol as: sty. Set Symbol Type (miSymbolTypeTrueTypeFont).

Third, you may use following propertyies to control the objects how to display on the maps:

\section{Style. Symbol Font Back Color property (Style object)}

Purpose: This contains the symbol background color and is used for symbol objects and can only apply to TrueType font symbols. This is an OLE_COLOR value. 


\section{Style. Symbol Font Color property (Style object)}

Purpose: Contains the symbol color. Used for symbol objects and can only apply to TrueType font symbols. This is an OLE_COLOR value.

\section{Style. Symbol Font Halo property (Style object)}

Purpose: This read/write property controls whether a halo is drawn around the symbol. Used for symbol objects and can only apply to TrueType font symbols. A halo is a 'buffer' around the symbol - to help offset it from the map beneath. This is a Boolean value that one can set to true for individual features.

\section{Style. Symbol Font Opaque property (Style object)}

Purpose: This read/write property controls whether the symbol displays a background color (and is thus opaque). Used for symbol objects and can only apply to TrueType font symbols. This is a Boolean value.

\section{Style. Symbol Font Rotation property (Style object)}

Purpose: Read-write integer value, indicating the number of degrees to rotate a TrueType symbol. Remarks This property should be set to an integer between zero and 360. Rotation is clockwise. This property only applies to TrueType font symbols, not to bitmap symbols; bitmap symbols cannot be rotated. Feature, Layer, and Theme objects support rotated symbols. If an object does not support rotated symbols, this property is ignored.

\section{Style. Symbol Font Shadow property (Style object)}

Purpose: Applies only to Layer Styles and Label Styles. Controls whether a shadow is drawn under the symbol. Used for symbol objects and can only apply to TrueType font symbols. This is a Boolean value.

\section{IMPLEMENTATION}

\section{A. Development Mode}

There are three modes such as independent development, simple redevelopment and integrated redevelopment in GIS project development.

Independent development means that your development is independent of any GIS platform and all works is implemented by developer self, such as spatial data collection, edit, analysis, processing and result output with visual C++, Delphi, $\mathrm{C}++$ Builder, YJ++ and so on. Some professional GIS developers are apt to develop plotting system by the way. These plotting systems is sophisticated plotting functions. But it is unfit to those projects which ask fulfill the development in given short time.

Simple redevelopment means that your development is hundred-percent dependent of certain redevelopment programming language with the GIS platform. Presently, business GIS development platforms such as ESRI ARCGIS, MapInfo Map GIS and Super Map GIS which are full-blown and well-known. These business GIS development platforms provide own macro languages. For example, ESRI ARCGIS provide with the Avenue, MapInfo professional provide with the Map Basic, and so on. Developers may make use of these macro languages to develop own plotting system on the GIS development platform. Although this mode is simple and effective, the performance of the macro languages is further more unadvanced than the advanced programming languages, so this mode is just suite for some simple systems.

Integrated redevelopment means that the developers make use of business GIS tool software or their Components to fulfill the basic functions of the plotting system. And using the third-party development platform, particular the visual development tool such as visual $\mathrm{C}++$, Delphi, visual Basic, Power Builder and so on, to fulfill integrated development, so as to implement the advanced plotting functions.

There are two ways for us to fulfill the integrated redevelopment, the one is OLE/DDE based development mode, the other is component based development mode. In COM based development mode, GIS control based development mode is more popular and effective than OLE/DDE based development mode and component based development mode. MapX is a tool for application developers. It offers the easiest, most cost-effective way to embed mapping functionality into new and existing applications. MapX is a DLL that can be quickly integrated into client applications using object-oriented languages such as Visual Basic, Delphi, and Visual C++. In the paper, the mode though MapX and both Visual C++ is used to plot regular unit symbol on map.

\section{B. Implement Process}

Before carry out the works in following text, you should have installed Visual Studio 2010 and MapX to the computer. The implement procedure is described below:

1. Create a new project by MFC AppWizard in Visual Studio 2010.

2. Adding the Map Control to the project. You will want to include MapX.cpp and MapX.h in your project. They contain the class definitions and method implementations for access to the MapX control. The MapX.h and MapX.cpp files can be found in the Samples50\CPP subdirectory where MapX is installed.

3. Creating a Map in the main form as Figure I. With MapX, it's easy to add a map to your application. In fact, you can add a working map to a Visual C++ form writing some single lines of code.

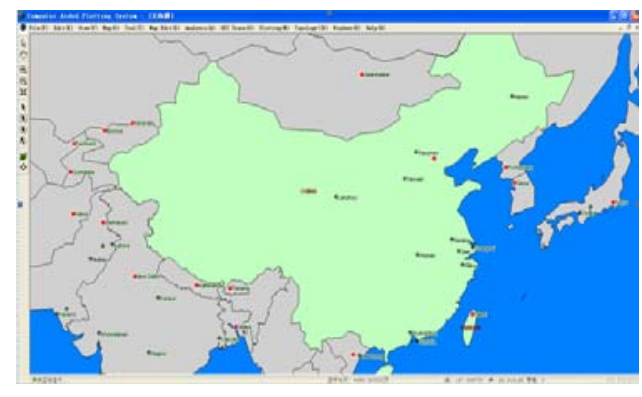

FIGURE I. A MAP IN THE MAIN FORM

4. Creating TrueType symbols by Font Creator, and export as the TrueType/OpenType font as a .ttf file. as Figure II 
5. Adding the TrueType symbols from the .ttf file to the given lay. as Figure III

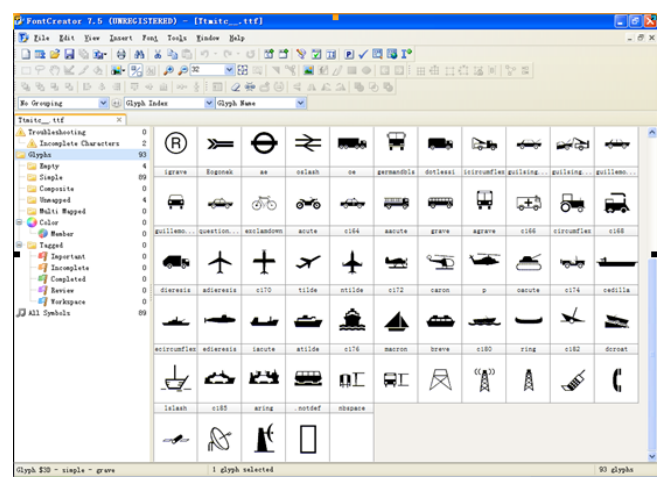

FIGURE II. THE TRUETYPE/OPENTYPE FONT IN A .TTF FILE

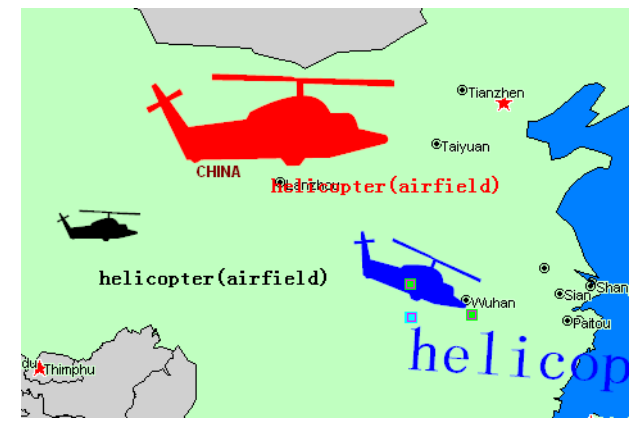

FIGURE III. THE FEATURES PLOTTING BASED ON THE TRUETYPE/OPENTYPE FONT

\section{CONCLUSIONS}

Using MapX TrueType font symbols, regular unit symbols are easy to be plotted on the map, and easy to be rotated, scaled up or scaled down. As the layers objects, the properties such as background color, symbol color, halo of the regular unit symbols may be controlled. It is showed that the method is effective and good practical effect.

\section{REFERENCES}

[1] MapX user guide. http://www.mapinfo.com/mapx/mapxdevguide.pdf.

[2] Li Y.-G,Design and Implementation of Navigation System Based on GIS Component MapX, JOURNAL OF COMPUTER AIDED DESIGN AND COMPUTER GRAPHICS, 1003-9775, 20010101

[3] Chuai Y.-y., Fan J.-z., Zhang X.-y.,Research and Realization of the Key-technology for the Development of WebGIS with MapX ,JOURNAL-LIAONING NORMAL UNIVERSITY NATURAL SCIENCE, 20050101

[4] Li Hai Feng, The Design of Campus Geographic Information System Based on MapX5.0+VB6.0, Key Engineering Materials,2012

[5] CONNECTMASTER AND MAPX AID BT GERMANY - Pitney Bowes MapInfo (now Software) and Dynamic Design team up with MapX and ConnectMaster respectively to help BT Germany manage its physical networks,GEO Connexion - Main Edition,2008 